\title{
Some Considerations on the IPFM Model for the Heart Rate Variability Analysis
}

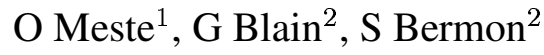 \\ ${ }^{1}$ University of Nice-Sophia Antipolis, Lab. I3S, France \\ ${ }^{2}$ University of Nice-Sophia Antipolis, Medical school, Lab. of Physiology, France
}

\begin{abstract}
The arch or inverted arch signals are characterizing patterns of the Respiratory Sinus Arrhytmia. The ability of any model in generating these patterns can be a good criteria for their selection. We show that using the tidal volume signal as the input of the model, an alternative to the IPFM can be proposed. The relevance of our model is shown with synthesized and real signals.
\end{abstract}

\section{Introduction}

It is commonly admitted that the IPFM model is a possible model for the generation of the $\mathrm{R}$ waves time occurrences $t_{k}$. The use of the outputs $t_{k} \mathrm{~s}$ for the frequency analysis of the heart rate variability has been studied in detail in several papers. The latest [1], [2] gives some recommendation in using these outputs in order to reduce or cancel the distortion of the spectrum. The problem of the choice for the model input is not usually addressed and is simplified using a single or a sum of cosines but not a real physiological signal, the respiration for instance. Since the Respiratory Sinus Arrhytmia (RSA) is one of the most important component of the heart rate variability it is straightforward that effort should be made in taking the respiration as an input of the model. In the last CinC we have proposed [3] a model for the generation of the $\mathrm{R}$ waves:

$$
\begin{array}{r}
\operatorname{ecg}(t)=\left(\cos \left(c_{1} t+c_{2} \cos \left(c_{3} t+c_{4}\right)+c_{5}\right)+1\right)^{200} \\
\text { with } c_{5} \in[0 ; \pi]
\end{array}
$$

where $c_{2} \cos \left(c_{3} t+c_{4}\right)$ is an external modulation. It will be shown in the following paper that similarly to the IPFM model a time occurrences generator, called PFM model, can be deduced from the expression (1). The condition for the correct use of the heart period signal defined as $h p(k)=$ $t_{k+1}-t_{k}$ will be also given. The aim of this communication is to compare these two models regarding their ability to mimic the RSA when a real or a synthetic respiration signal is used as the input of the model.
It has been shown that the repetitive pattern of the RSA can exhibit different shapes [4] depending on the subjects and experimental conditions. These shapes could be compared to arch or inverted arch and will be designated consequently. Since this shape analysis is a step forward compared to classical frequency analysis we will check whether the PFM or the IPFM can exhibit these patterns under some artificial experimental conditions. We will close this communication presenting a real case. This case compares the outputs obtained from the two models, using the recorded respiration volume as the input, to the measured heart period signal from the real ECG signal.

\section{Models definitions and properties}

Starting from the R-waves generator defined by (1) and assuming that the maximum of $\operatorname{ecg}(t)$ correspond to the time occurrence of interest $t_{k}$, it is clear that the $t_{k} \mathrm{~s}$ are the solution of the equation:

$$
c_{1} t_{k}+c_{2} \cos \left(c_{3} t_{k}+c_{4}\right)+c_{5}=2 \pi k
$$

with $k=0 \ldots K$. The proposed solution of this equation is:

$$
t_{k}=\frac{2 \pi}{c_{1}} k-\frac{c_{2}}{c_{1}} \cos \left(\frac{c_{3}}{c_{1}} 2 \pi k+c_{4}-\frac{c_{3} c_{5}}{c_{1}}\right)-\frac{c_{5}}{c_{1}}
$$

This is an approximated solution in the simple case that the modulating function is a single cosine. It can be shown that it is an acceptable solution when the condition:

$$
\frac{c_{2} c_{3}}{c_{1}} \ll \pi
$$

is verified. From (3) the heart period signal can be calculated by $h p(k)=t_{k+1}-t_{k}$ and is finally:

$h p(k)=\frac{2 \pi}{c_{1}}+2 \sin \left(\frac{c_{3}}{c_{1}} \pi\right) \frac{c_{2}}{c_{1}} \sin \left(\frac{c_{3}}{c_{1}} 2 \pi k+\frac{c_{3}}{c_{1}} \pi+c_{4}-\frac{c_{3} c_{5}}{c_{1}}\right)$

We can state that if the model proposed in (1) synthesize a real ECG (R-waves), the deduced $h p(k)$ is a cosine 
function uniformly sampled whose reduced pulsation is $\frac{c_{3}}{c_{1}} 2 \pi$ with a magnitude equal to $2 \sin \left(\frac{c_{3}}{c_{1}} \pi\right) \frac{c_{2}}{c_{1}}$ added to an offset corresponding to the mean heart period $T$. This permit us to establish the relation $c_{1}=2 \pi / T$. When the quantity $T$ is expressed in seconds, it plays the role of the sampling period giving the relation $c_{3}=2 \pi f_{v}$ with $f_{v}$ the frequency of the variability expressed in Hertz. In summary, introducing $T$ as the mean heart period, $f_{v}$ the frequency of the variability and $A$ the magnitude of the variations, we get the equalities:

$$
\left\{\begin{array}{c}
c_{1}=\frac{2 \pi}{T} \\
c_{2}=\frac{A \pi}{T \sin \left(T f_{v} \pi\right)} \\
c_{3}=2 \pi f_{v}
\end{array}\right.
$$

The validity condition (4) is then

$$
\frac{A f_{v}}{\sin \left(\pi T f_{v}\right)} \ll 1
$$

From (6), we conclude that the magnitude $A$ of the $h p(k)$ variability is a function of the magnitude $c_{2}$ of the modulation, the mean heart period $T$ and the frequency of the variability $f_{v}$ and is given by:

$$
A=\frac{T c_{2}}{\pi} \sin \left(f_{v} T \pi\right)
$$

In fact, the modulation function is $m_{1}(t)=c_{2} \cos \left(c_{3} t+c_{4}\right)$ and appears in (2) using (8) as:

$$
k T=t_{k}+\frac{T}{2 \pi} m_{1}\left(t_{k}\right)+\frac{T}{2 \pi} c_{5}
$$

This equation is similar than those from the IPFM model [1] which is:

$$
k T=t_{k}+\int_{0}^{t_{k}} m_{2}(t) d t
$$

Since on the contrary to (10) the modulation function is not integrated in (9) we will call PFM model the expression (9).

When choosing for the modulation function in (10) the expression:

$$
m_{2}(t)=a_{2} \sin \left(a_{3} t+a_{4}\right)
$$

it can be shown that the corresponding magnitude of the variability under the IPFM model assumption is:

$$
A=-\frac{a_{2}}{\pi f_{v}} \sin \left(f_{v} T \pi\right)
$$

This result can be compared to (8) where the PFM model is used. It is clear that these quantities strongly differ and will help us to understand the results from the simulation section. This difference is certainly important under dynamic conditions where the mean heart period $T$ is considered as time varying. This more realistic condition can be addressed using the PFM model since replacing $c_{1}$ by $c_{1}(t)$ in (1) gives the equation:

$t_{k}=\frac{2 \pi}{c_{1}\left(t_{k}\right)} k-\frac{c_{2}}{c_{1}\left(t_{k}\right)} \cos \left(\frac{c_{3}}{c_{1}\left(t_{k}\right)} 2 \pi k+c_{4}-\frac{c_{3} c_{5}}{c_{1}\left(t_{k}\right)}\right)-\frac{c_{5}}{c_{1}\left(t_{k}\right)}$

The calculation of $h p(k)$ from (13) is tedious and can be achieved using the instantaneous pulsation $\beta_{1}\left(t_{k}\right)$ defined by:

$$
\beta_{1}\left(t_{k}\right)=\left.\frac{d}{d t}\left(t c_{1}(t)\right)\right|_{t=t_{k}}
$$

Which is approximated by:

$$
\beta_{1}\left(t_{k}\right) \approx \frac{t_{k+1} c_{1}\left(t_{k+1}\right)-t_{k} c_{1}\left(t_{k}\right)}{t_{k+1}-t_{k}}
$$

Since the definition of $h p(k)$ is $h p(k)=t_{k+1}-t_{k}$, we deduce from (15):

$$
h p(k)=\frac{1}{\beta_{1}\left(t_{k}\right)}\left[t_{k+1} c_{1}\left(t_{k+1}\right)-t_{k} c_{1}\left(t_{k}\right)\right]
$$

After some simplifications we finally get:

$$
\begin{array}{r}
h p(k) \approx \frac{2 \pi}{\beta_{1}\left(t_{k}\right)}+2 \frac{c_{2}}{\beta_{1}\left(t_{k}\right)} \sin \left(\frac{\pi c_{3}}{\beta_{1}\left(t_{k}\right)}\right) \\
\quad \sin \left(\frac{c_{3} 2 \pi k}{c_{1}\left(t_{k}\right)}+\frac{c_{3} \pi}{c_{1}\left(t_{k}\right)}+c_{4}-\frac{c_{3} c_{5}}{c_{1}\left(t_{k}\right)}\right)
\end{array}
$$

leading to a definition of the "instantaneous" mean heart period $T\left(t_{k}\right)$ :

$$
T\left(t_{k}\right)=\frac{2 \pi}{\beta_{1}\left(t_{k}\right)}
$$

From this result we can conclude that on the contrary to the "instantaneous" mean heart period which is a function of the instantaneous pulsation $\beta_{1}\left(t_{k}\right)$, the time varying sampling frequency is a function of $c_{1}\left(t_{k}\right)$ what is unexpected. It means that when the time varying mean heart period is estimated from the real HP signal, using a low-order polynomial fitting for instance, the frequency of the variability should not be directly converted from the quantity $T\left(t_{k}\right)[3]$.

We have shown in this section that a new modelling of the heart period can be proposed from the PFM model. The results differ from the IPFM model outputs concerning the magnitude of the variability but also the phase. In order to illustrate these differences we are going to compare the two approaches in simulation and real cases.

\section{Simulations with the arch signal}

In [4], a non linear approach has been proposed in order to analyze R-R interval time series. These authors have noticed that depending of the patient, the clustering of the 
heartbeat could be either at long or short R-R intervals. These two patterns will be called arch or inverted arch signal because of the global shape. Since the output of the competing models is expected to exhibit the RSA, the input will be assumed to be the tidal volume. Then, prior to simulate the ability of the models to generate the arch signals, we are going to calculate the output of the models when the input is a simulated tidal volume signal when the condition (7) is fulfilled. The figure 1 shows two kinds of synthetic tidal signals with a ratio of expiration to inspiration length at about (4:3). The tidal volume signal has been synthesized in two different manners in order to illustrate the possible distortion in recording this quantity using a respiratory inductive plethysmograph [5]. These

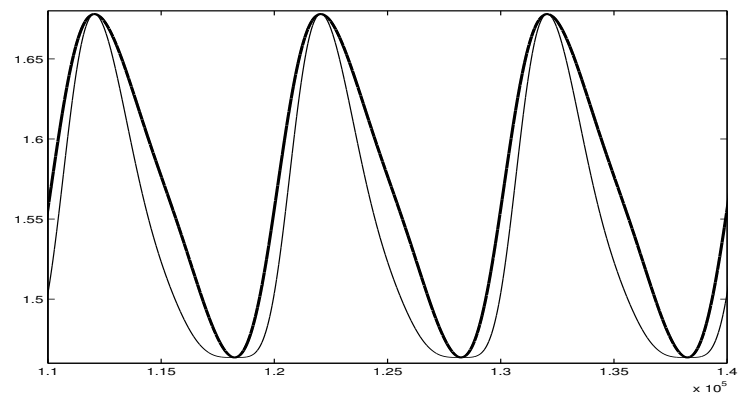

Figure 1. Two synthesized tidal volume signals

two modulation functions has been used in the PFM (9) and IPFM (10) models yielding the simulated $m\left(t_{k}\right)$ given in Fig. 2 and 3, respectively. From these results we can

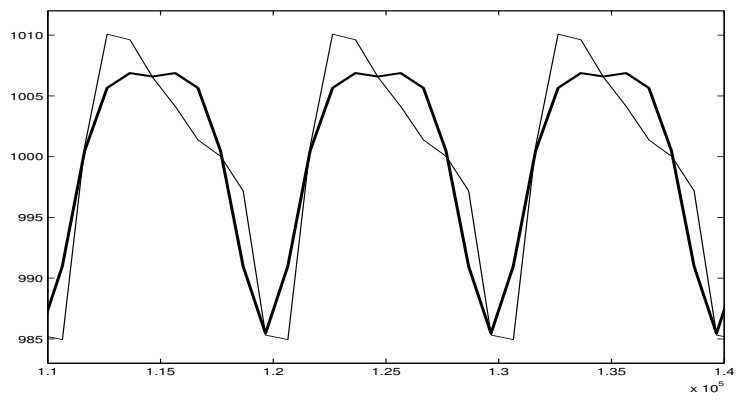

Figure 2. The heart period signals from the PFM model function of time for the two tidal volume signals

remark that the PFM model is more sensitive to the shape of the input and it is clear that the output of the IPFM model doesn't exhibit the classical RSA pattern, i.e. the duration of the increase of the heart rate should be longer than the decrease. Since the aim of this simulation is to reproduce a paced respiration condition, it is noticeable to see that the phase of the signals in Fig. 2 is in agreement with real cases regards to the tidal volume signal in Fig. 1, on the contrary to the IPFM model.

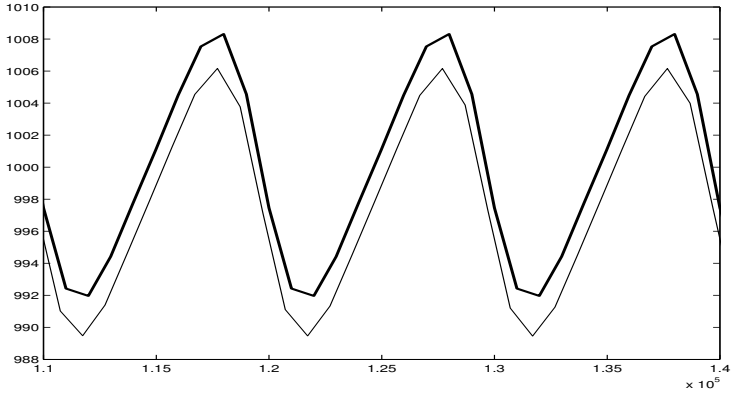

Figure 3. The heart period signals from the IPFM model function of time for the two tidal volume signals

The second simulation concerns the ability to produce arch signals observed in real cases. As in [4], we simulate the $h p$ signal when the condition (7) is not fulfilled. In Fig. 4 two kinds of simulated tidal volumes are shown with ratios of (1:1) (thick line for the pure sinusoid) and (4:3) (thin line). The $h p$ results from the PFM model clearly

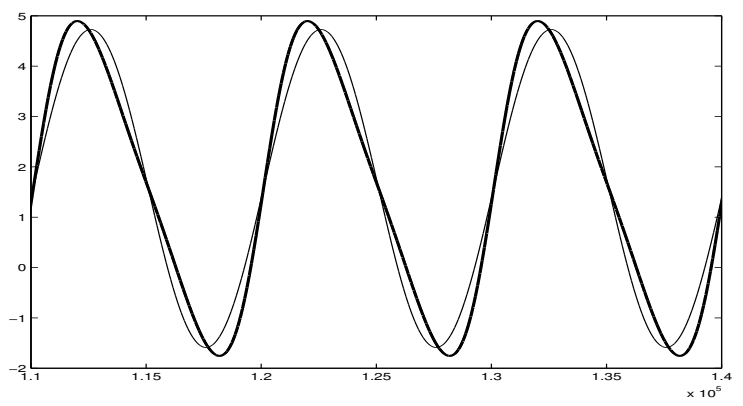

Figure 4. The symmetric (thin line) and asymmetric (thick line) synthesized tidal volume inputs

exhibit the inverse arch signal in Fig. 5 for the pure sinusoid as mentioned in [1] and the arch signal in Fig. 6 when the simulated tidal volume signal is not symmetric. Under the

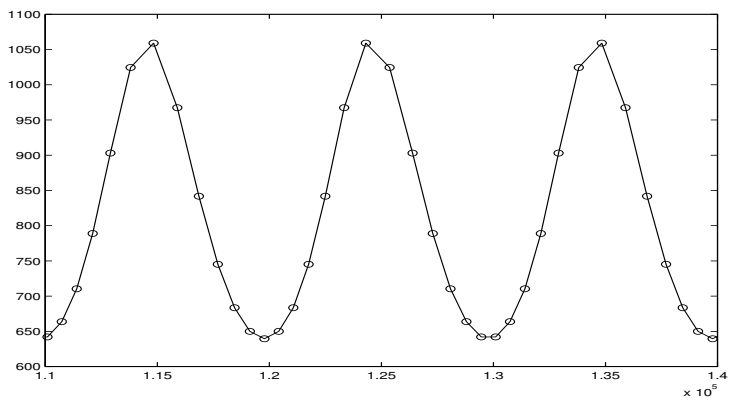

Figure 5. The heart period signal (inverse arch like shape) from the PFM model function of time for the symmetric (1:1) tidal volume signal

condition that the PFM model is a valid one, the observed patterns of the RSA could be simply explained introducing 


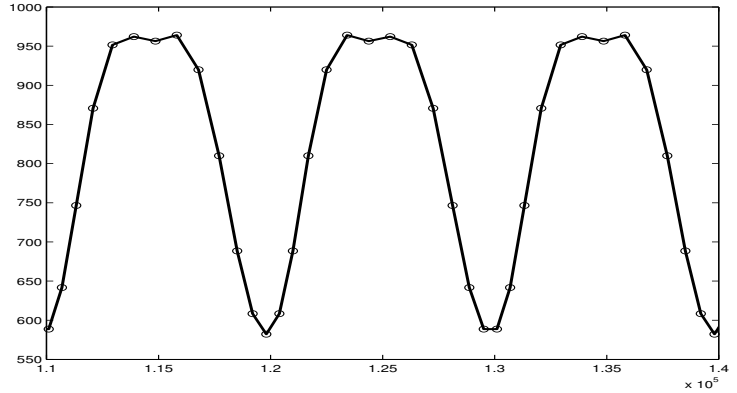

Figure 6. The heart period signal (arch like shape) from the PFM model function of time for the asymmetric (4:3) tidal volume signal

the effect of the variation of tidal volume shape or ratio.

\section{A real case}

The ECG signal has been recorded during a paced respiration $(0.1 \mathrm{~Hz})$ session in the supine position. The tidal volume has been acquired through an uncalibrated respiratory inductive plethysmograph and is shown in Fig. 7. Note that this signal has been enhanced in order to be used as the modulating function in (1) and after choosing a constant mean heart period equal to 1.07 seconds. The HP signal from the PFM model can be compared to the real one and the output of the IPFM model in Fig. 8 where the good matching between the real HP and those from the PFM model can be observed. However a small delay can be seen in the decreasing interval of the heart period which could be caused by the distortion in the measurement of the real tidal volume. This distortion effect has been simulated in Fig. 2.

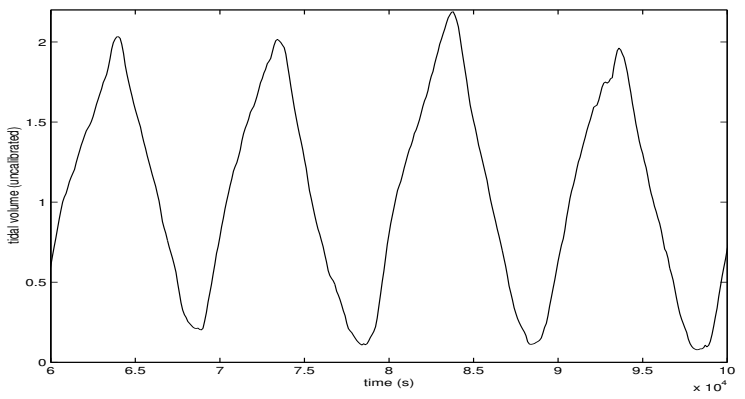

Figure 7. Real tidal volume recorded using an uncalibrated respiratory inductive plethysmograph

\section{Conclusion}

We have proposed a model for the generation of the ECG ( $\mathrm{R}$ waves) and the derivation giving the heart period from

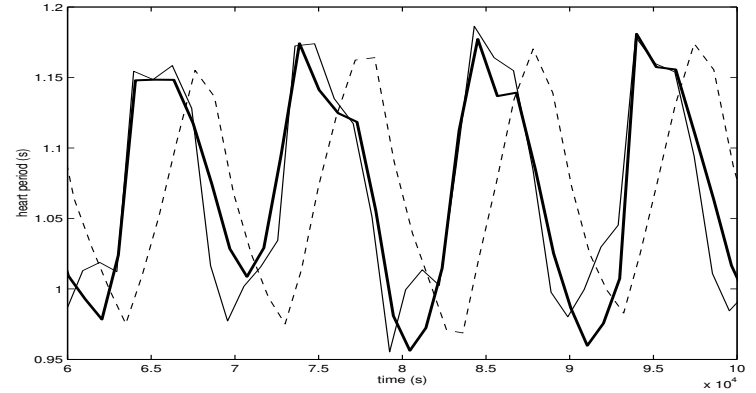

Figure 8. The real HP signal (thick solid line), the HP signal from the PFM model (thin solid line), the HP signal from the IPFM model (dashed line)

the time of occurrence of the waves. Using the arch signal characterizing the RSA, we have shown that our model could be a good candidate for the modelling of the heart period variability under paced respiration condition. This has been achieved in simulation but also on a real case.

\section{References}

[1] Mateo J, Laguna P. Improved heart rate variability signal analysis from the beat occurence times according to the ipfm model. IEEE Trans Biomed Eng 2000;47(8):985-996.

[2] Brennan M, Malaniswami M, Kamen P. Distortion properties of the interval spectrum of ipfm generated heartbeats for the heart rate variability analysis. IEEE Trans Biomed Eng 2001; 48(11):1251-1264.

[3] Meste O, Blain G, Bermon S. Analysis of the respiratory and cardiac systems coupling in pyramidal exercise using a timevarying model. In 29th annual conference on Computers in Cardiology. Memphis, USA, September 2002; .

[4] Suder K, Drepper FR, Schiek M, Abel HH. One-dimensional, nonlinear determinism characterizes heart rate pattern during paced respiration. Am J Physiol 1998;H1092-H1102.

[5] Gothberg S, Parker TA, Griebel J, Abman SH, Kinsella JP. Lung volume recruitment in lambs during highfrequency oscillatory ventilation using respiratory inductive plethysmography. Pediatric Research 2001;49(1):38-44.

\section{Acknowledgments}

The authors acknowledge Paul Nolan, Redmond Shouldice, Conor Heneghan, and Philip Nolan of University College Dublin/St. Vincent's University Hospital, Dublin, Ireland for provision of ECG and respiratory data

Address for correspondence:

Olivier Meste, Lab. I3S, UNSA-CNRS,2000, route de Lucioles. BP 121, 06903 Sophia Antipolis Cedex, France. tel./fax: +3304 92965104 / +330492965155

meste@i3s.unice.fr 